\title{
PREMIERS RÉSULTATS DE TESTS POUR L'ÉVALUATION DE L'EFFICACITÉ D'UN INSECTICIDE SYSTÉMIQUE CONTRE L'HYLOBE (Hylobius abietis L., Coll. Curculionidae)
}

\author{
G. LEMPERIÈRE - J.-M. JULIEN
}

Le Grand Charançon du Pin, Hylobius abietis L., est le ravageur le plus dommageable aux jeunes plantations de résineux. Les pullulations de cet insecte sont particulièrement spectaculaires sur les jeunes reboisements d'Épicéas, de Pins et de Douglas effectués après des coupes rases de Pins ou d'Épicéas. Cet insecte se multiplie en effet dans les souches ou les bûches laissées sur place après l'exploitation. Les morsures de nutrition sur l'écorce des jeunes plants provoquent ensuite un rapide dépérissement. Les dégâts ont lieu au collet et sur la tige des jeunes arbres qui sont écorcés en quelques jours. Deux périodes d'attaque peuvent être distinguées :

- au printemps, de mi-avril à mi-juin, avec des adultes ayant hiverné dans la litière qui doivent reconstituer leurs réserves pour la ponte :

- à la fin de l'été, avec ces mêmes adultes et les adultes de la génération suivante issus de larves ayant hiverné, voire de pontes du printemps.

La densité des populations d'Hylobe peut atteindre des proportions telles que les plantations sont parfois détruites à cent pour cent.

Dégâts du Grand Charançon du Pin sur un jeune plant de Douglas.

Photo Service régional de la Protection des végétaux de Beaune.

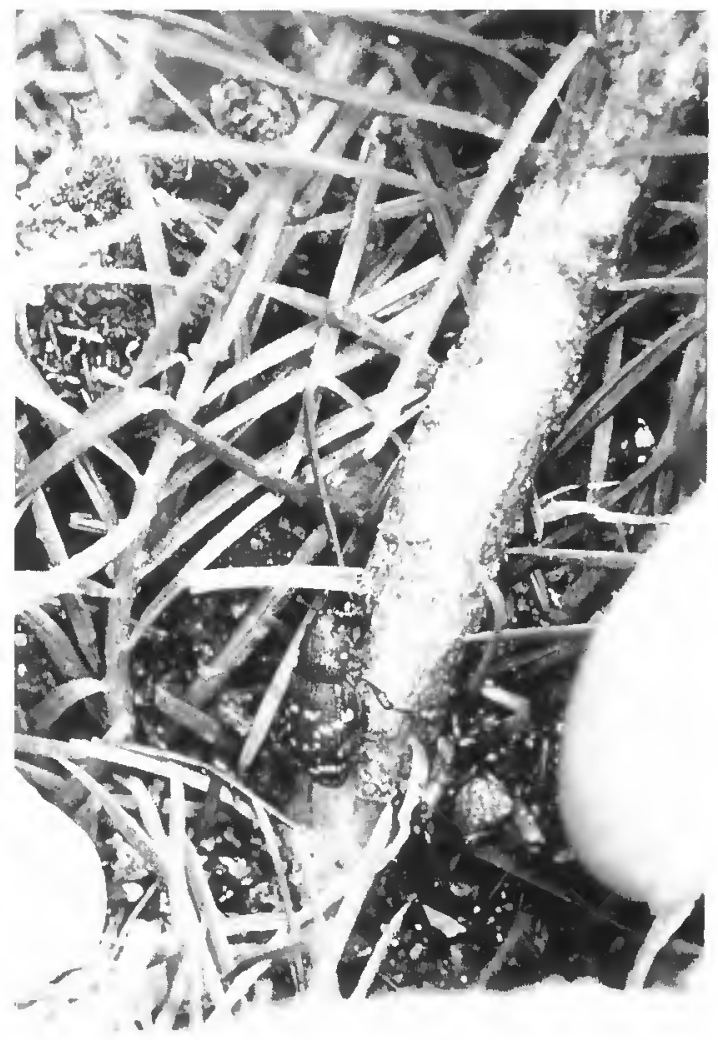




\section{G. LEMPERIÈRE - J.-M. JULIEN}

Différentes méthodes de lutte ont donc été employées afin de limiter les dommages à un seuil tolérable de 5 à $10 \%$ de perte, comparable à la mortalité naturelle constatée à la replantation.

C'est ainsi que, jusqu'à la fin des années soixante, le traitement courant consistait en un trempage ou une pulvérisation dans une solution de Dichlorodiphényltrichloréthane (DDT) à $5 \%$. Ce procédé fut ensuite remplacé par un traitement à base de Lindane, toujours en usage de nos jours, aussi bien en trempage qu'en pulvérisation.

II est aussi intéressant de noter que, jusque récemment, les différents tests menés tant en laboratoire qu'en plein champ, utilisaient des insecticides dont les caractéristiques (toxicité visà-vis de l'insecte, facilité d'emploi, rémanence,...) tenaient peu compte de l'impact sur l'environnement.

Citons pour mémoire l'emploi du Méthidathion et de l'Aldicarbe, tous deux classés au tableau A, notés comme dangereux ou extrêmement dangereux pour les arthropodes auxiliaires, les abeilles, le gibier et les poissons. Les métabolites toxiques de l'Aldicarbe (sulfones et sulfoxydes) sont d'ailleurs à l'origine de la pollution de nappes phréatiques aux États-Unis (Garner et al., 1986).

Des méthodes de protection mécanique des plants ont aussi été employées (Teno collar, chaussettes suédoises) avec des résultats très variables.

II paraissait donc intéressant de tester un insecticide possédant un pouvoir toxique et répulsif vis-à-vis de l'insecte, le but final étant la protection du plant. Cet insecticide devait en outre assurer une protection suffisamment prolongée permettant d'éviter la répétition de traitements des parties aériennes, comme c'est actuellement le cas, ou le trempage des plants avant transplantation. Enfin le produit employé devait être sans danger pour l'environnement.

L'opportunité nous a été donnée par la firme australienne Incitec International d'utiliser une nouvelle technologie qui a déjà fait ses preuves dans la protection de cultures contre divers insectes en Australie, notamment Lepidiota spp., Anomala spp., Macrotermes spp., Coptotermes spp. sur canne à sucre (May et Boehm, 1986 ; Plowman et O'Hanlon, 1984).

Nous avons ainsi, à partir du printemps 1987, réalisé des tests au laboratoire et en plein champ, avec un insecticide systémique (Carbosulfan), conditionné sous forme de granulés comprenant une matrice en polymère au sein de laquelle se trouve la matière active. La libération progressive du produit est guidée par le taux d'humidité du sol et peut se prolonger pendant 120 semaines.

\section{ESSAIS EN LABORATOIRE}

\section{Matériel et méthodes}

\section{- Matériel végétal}

Outre un témoin de 20 plants de Sapin de Douglas, nos essais en laboratoire ont porté sur des lots de 20 à 40 plants, âgés de trois à quatre ans de quatre espèces forestières fréquemment utilisées dans les reboisements en résineux: le Sapin de Douglas (Pseudotsuga douglasii Carr.), l'Épicéa commun (Picea excelsa Link.), le Pin sylvestre (Pinus sylvestris L.), le Pin laricio de Corse (Pinus nigra Arn. ssp. laricio Poir.). Ces plants ont été mis en place en pots de deux litres.

\section{- Dasage et application des produits}

L'insecticide utilisé est du Carbosulfan (famille des carbamates) produit par FMC et formulé par Incitec International sous le nom de Marshal Suxon. 
Pour chacune des essences, quatre doses ont èté employées, à savoir 2, 4, 8 et 16 grammes de granulés correspondant à $0,2-0,4-0,8$ et $1,6 \mathrm{~g}$ de matière active. L'incorporation des granulés dans le sol a été effectuée simultanèment à la plantation des jeunes résineux dans les pots, l'insecticide se trouvant ainsi en contact avec les racines.

À noter que des tests avec des insecticides systémiques (notamment le Phorate) ont déjà été menés dans d'autres pays (Bakke, 1971 ; King, 1978 non publiẻ) et ont donné des résultats intéressants avec néanmoins l'apparition de phénomènes de phytotoxicitè.

\section{- Matériel animal}

Pour les besoins des essais, nous avons récolté, au printemps, sur des rémanents de coupes, des adultes d'Hylobes qui ont été isolés et placés au réfrigérateur sans nourriture, 48 heures avant les tests. Ces Hylobes étaient issus de la génération d'adultes hivernants de 1986-87.

\section{- Exécution des essais}

Les insectes ont été mis en présence des jeunes plants 30 jours après le traitement de ceux-ci, afin de s'assurer que les plants avaient bien absorbé l'insecticide. Les essais ont eu lieu dans une salle maintenue à une température constante de $19^{\circ} \mathrm{C}$. Les insectes des deux sexes étaient répartis à raison d'un insecte par plant ; chaque plant était recouvert d'une toile ne permettant pas à l'insecte de s'échapper.

En plus des essais en pots, un essai avec des rameaux de plants de Douglas traitès (lot traité à $0,4 \mathrm{~g}$ de matière active) a été mené sur 20 insectes selon le protocole utilisé par Delorme et Malphettes (1976).

Les contrôles ont portè à la fois sur la mortalité des insectes et l'intensité des dommages sur les plants. Ils ont été effectués un, deux, quatre et sept jours après la mise en place des insectes.

Après rècolte, les insectes encore vivants ont été testés dans une coupelle de bois de $10 \mathrm{~cm}$ de diamètre, placée sous une lampe. Ils ont été classés comme morts lorsqu'ils étaient incapables de sortir de la coupelle en une demi-heure (critères utilisés par Delorme et Malphettes, op. cité).

L'intensité des dommages a èté relevée selon une échelle de notation des attaques utilisée par le CEMAGREF (division Protection de la Nature, 1985) pour des essais de terrain. Cette même échelle de notation, légèrement modifiée, a étė employée pour nos relevés de terrain. Elle se compose comme suit :

$-0=$ pas de dommages.

$-1=$ morsures individualisées ( 1 à 5 ).

$-2=5$ à 10 morsures individualisées.

$-3=$ plus de 10 morsures, quelques coalescences.

$-4=$ nombreuses coalescences, épiderme fortement attaqué.

$-5=$ mort du plant par ceinturage dû aux morsures d'Hylobe.

Bien que faite pour le Douglas, elle est valable pour les autres essences.

\section{- Résultats et perspectives}

Remarque préliminaire concernant l'analyse.

Si différents tableaux de données peuvent être analysés selon la méthode classique du test $\chi^{\mathrm{e}}$, un certain nombre d'entre eux ne suivent pas la règle de Cochran; dans certains cas, la moitié 


\section{G. LEMPERIĖRE - J.-M. JULIEN}

Tableau 1 Mortalite des insectes observée 1, 2, 4 et 7 jours après mise en place de l'expérience (en pourcentages)

\begin{tabular}{|c|c|c|c|c|c|}
\hline Espèce & $\begin{array}{c}\text { Dose } \\
\text { (grammes de } \\
\text { matière active) }\end{array}$ & $\begin{array}{l}\text { Après } 1 \text { jour } \\
\qquad(\%)\end{array}$ & $\begin{array}{l}\text { Après } 2 \text { jours } \\
\qquad(\%)\end{array}$ & $\begin{array}{l}\text { Après } 4 \text { jours } \\
(\%)\end{array}$ & $\begin{array}{c}\text { Après } 7 \text { jours } \\
(\%)\end{array}$ \\
\hline $\begin{array}{l}\text { Sapin de Douglas } \\
(2+1) \ldots \ldots \ldots \ldots \ldots\end{array}$ & $\begin{array}{l}0,2 \\
0,4 \\
0,8 \\
1,6\end{array}$ & $\begin{array}{r}0 \\
0 \\
20 \\
0\end{array}$ & $\begin{array}{r}0 \\
0 \\
40 \\
0\end{array}$ & $\begin{array}{r}20 \\
0 \\
40 \\
20\end{array}$ & $\begin{array}{l}60 \\
60 \\
80 \\
60\end{array}$ \\
\hline $\begin{array}{l}\text { Sapin de Douglas } \\
(2+2) \ldots \ldots \ldots \ldots\end{array}$ & $\begin{array}{l}0,2 \\
0,4 \\
0,8 \\
1,6\end{array}$ & $\begin{array}{r}0 \\
20 \\
0 \\
0\end{array}$ & $\begin{array}{r}0 \\
20 \\
0 \\
0\end{array}$ & $\begin{array}{r}0 \\
40 \\
0 \\
20\end{array}$ & $\begin{array}{l}80 \\
60 \\
60 \\
80\end{array}$ \\
\hline $\begin{array}{l}\text { Épicéa commun } \\
(2+1) \ldots \ldots \ldots\end{array}$ & $\begin{array}{l}0,2 \\
0,4 \\
0,8 \\
1,6\end{array}$ & $\begin{array}{r}20 \\
0 \\
20 \\
20\end{array}$ & $\begin{array}{r}20 \\
0 \\
20 \\
20\end{array}$ & $\begin{array}{l}20 \\
20 \\
60 \\
20\end{array}$ & $\begin{array}{r}80 \\
80 \\
100 \\
80\end{array}$ \\
\hline $\begin{array}{l}\text { Épicéa commun } \\
(2+2) \ldots \ldots \ldots\end{array}$ & $\begin{array}{l}0,2 \\
0,4 \\
0,8 \\
1,6\end{array}$ & $\begin{array}{r}40 \\
0 \\
0 \\
0\end{array}$ & $\begin{array}{r}40 \\
0 \\
0 \\
0\end{array}$ & $\begin{array}{r}40 \\
0 \\
20 \\
20\end{array}$ & $\begin{array}{r}80 \\
100 \\
20 \\
20\end{array}$ \\
\hline Pin sylvestre & $\begin{array}{l}0,2 \\
0,4 \\
0,8 \\
1,6\end{array}$ & $\begin{array}{l}10 \\
20 \\
40 \\
20\end{array}$ & $\begin{array}{l}10 \\
20 \\
80 \\
30\end{array}$ & $\begin{array}{l}40 \\
40 \\
80 \\
60\end{array}$ & $\begin{array}{l}50 \\
50 \\
80 \\
70\end{array}$ \\
\hline Pin laricio $\ldots . .$. & $\begin{array}{l}0,2 \\
0,4 \\
0,8 \\
1,6\end{array}$ & $\begin{array}{r}0 \\
0 \\
0 \\
10\end{array}$ & $\begin{array}{r}20 \\
0 \\
40 \\
40\end{array}$ & $\begin{array}{l}50 \\
20 \\
50 \\
40\end{array}$ & $\begin{array}{l}70 \\
40 \\
90 \\
40\end{array}$ \\
\hline Sapin de Douglas ... & Témoin & 0 & 0 & 0 & 0 \\
\hline
\end{tabular}

Comparaison entre espéces : test de Kruskall Wallis; $H=5,13>11,07=$ valeur critique pour 0,$05 ;$ pas d'hètérogénéité. Comparaison entre doses : test de Kruskall Wallis; $\mathrm{H}=3,28<7,8=$ valeur critique pour 0,$05 ;$ pas d'effet de dose.

Tableau II Observations des dégâts sur les plants, 7 jours après mise en place de l'expérience

\begin{tabular}{|c|c|c|c|c|c|c|c|}
\hline \multicolumn{7}{|c|}{ Nombre d'arbres dans la classe } \\
\hline $\begin{array}{c}\text { Notation } \\
\text { des dégâts }\end{array}$ & Témoin & $\begin{array}{c}\text { Douglas } \\
(2+1)\end{array}$ & $\begin{array}{c}\text { Douglas } \\
(2+2)\end{array}$ & $\begin{array}{c}\text { Épicéa } \\
(2+1)\end{array}$ & $\begin{array}{c}\text { Épicea } \\
(2+2)\end{array}$ & $\begin{array}{c}\text { Pin } \\
\text { sylvestre }\end{array}$ & Pin laricio \\
\hline \multirow{2}{*}{0} & 1 & 4 & 1 & 5 & 9 & 10 & 13 \\
1 & 2 & 12 & 14 & 15 & 11 & 30 & 27 \\
2 & 6 & 4 & 3 & 0 & 0 & 0 & 0 \\
3 & 10 & 0 & 2 & 0 & 0 & 0 & 0 \\
4 & 1 & 0 & 0 & 0 & 0 & 0 & 0 \\
5 & 0 & 0 & 0 & 0 & 0 & 0 & 0 \\
\cline { 2 - 9 } & 20 & 20 & 20 & 20 & 20 & 40 & 40 \\
\hline
\end{tabular}

Comparaison entre espêces : test de Kruskall Wallis; $H=5,93<\uparrow 1,07$ valeur critique pour 0,05 ; pas d'héterogènèitè. Comparaison entre doses : test de Kruskall Wallis; $\mathrm{H}=5,36<7,8$ valeur critique pour 0,05 ; pas d'hétèrogènéite.

Separation témoin-traitement: test de Wilcoxon Mann Whitney; $Z=6,78>1,64$; sèparation entre tèmoin et traitements. 
des fréquences attendues est inférieure à 5 . S'il est toujours possible d'opérer des regroupements, cette opération risque parfois de gommer trop d'informations et d'aboutir à des résultats incorrects. Comme de plus les données sont de nature semi-quantitative, et que les conditions de normalité ne sont pas vérifiées, les tests de comparaison non paramétriques sont la statistique appropriée.

Observations sur la mortalité des insectes

Le tableau I (page 414) fait ètat des mortalités en fonction des doses employées et de différentes essences forestières testées. La simple lecture du tableau I montre une séparation entre pots traitès et pots tẻmoins. Par ailleurs, la comparaison entre espèces ne montre pas d'hétérogénéité entre ces groupes. La comparaison entre les doses ne montre aucun effet de dose.

Observations des dégâts sur les plants

À partir du tableau II (page 414), le test de Wilcoxon Mann Whitney montre une séparation entre pots traités et pots témoins. La comparaison entre les espèces ne montre pas d'hétérogénéité entre ces groupes. La comparaison entre les doses ne montre aucun effet de dose. Ces résultats sont conformes à ceux obtenus avec les mortalités. L'étude par comparaisons multiples des différentes espèces indique que c'est le groupe témoin qui est responsable de l'hétérogénéité.

De nouveaux essais sur plants en pots ont été menés en 1988 avec le Douglas et les mêmes doses de traitement. Les insectes provenaient d'élevage sur milieu semi-artificiel (Lemperière, 1984) et ont été testés à raison de 4 individus par plant. II semble exister en effet sur le terrain un processus d'agrègation des insectes en réponse aux substances volatiles èmises par le vègétal-hôte lorsque les insectes pratiquent leurs morsures de nutrition (Tilles et al., 1986). Les insectes ont été mis en présence des plants sept jours après le traitement au Carbosulfan. Aucune différence significative n'a pu être notée entre les plants traités et les témoins. Une période minimale d'une dizaine de jours paraît nécessaire pour la migration du produit dans les plants et le début de leur protection.

\section{ESSAIS DE TERRAIN}

\section{Description des sites}

Les essais de terrain ont èté menés sur deux sites situés en région Limousin, dans le département de la Corrèze:

- Site 1: Lamazière-Basse, plantation de Douglas $(2+2)$ située à une altitude de $900 \mathrm{~m}$, d'une surface de 12 ha, sur une coupe rase de Pins sylvestres effectuée durant I'hiver 1985-86, donc un an avant l'essai ; le sol est de type brun acide et a été travaillé après la coupe rase.

- Site 2: Pérols-sur-Vézère, plantation d'Épicéas et de Douglas $(2+2)$ situèe à une altitude de $600 \mathrm{~m}$, d'une surface de 10 ha, sur une coupe rase de Pins sylvestres de l'hiver 198687 , donc quelques mois avant l'essai ; le sol est de même nature qu'à Lamazière et a également èté travaillè.

\section{Nature des essais}

Chacun des traitements sur les deux sites a été effectué à la plantation, le 14 avril 1987. Les granulés ont été appliqués dans le fond des trous de plantation, au contact des racines et des plants. Trois doses ont été employées à savoir 2,4 et 8 grammes de granulés à $10 \%$ de matière active. 


\section{G. LEMPERIÈRE - J.-M. JULIEN}

Les dispositifs expérimentaux sur les deux sites consistent en trois blocs de 50 plants chacun traités aux trois doses, et trois blocs témoins de 50 plants non traités.

Le reste de la plantation a subi un traitement classique à base de Lindane.

\section{Résultats}

\section{- Lamazière-Basse}

En 1987, le site a fait l'objet de sept relevés et l'analyse des résultats a porté sur les cinq premiers. En effet, nous n'avons notè aucune évolution des dégâts entre le 22 août et le 20 novembre, date du dernier relevé. Lors de l'installation du dispositif expérimental, une forte attaque d'Hylobes a été observée sur l'ensemble du chantier de reboisement.

Les résultats sont consignés dans les tableaux III, IV, V, VI (p. 416 à 419). Le test de Wilcoxon Mann Whitney différencie les parcelles témoins des parcelles traitées quelle que soit la date du

Tableau III Bilan des dégâts sur la parcelle expérimentale de Lamazière-Basse (19) 1987

\begin{tabular}{|c|c|c|c|c|c|c|c|c|c|c|c|}
\hline \multirow{3}{*}{$\begin{array}{l}\text { Date de } \\
\text { relevé }\end{array}$} & \multirow{3}{*}{$\begin{array}{l}\text { Classes } \\
\text { de dégâts }\end{array}$} & \multicolumn{6}{|c|}{ Nombre d'arbres dans la classe } & \multirow{2}{*}{\multicolumn{2}{|c|}{$\begin{array}{l}\text { Test 1: } \\
\text { Comparaison des parcelles }\end{array}$}} & \multirow{2}{*}{\multicolumn{2}{|c|}{$\begin{array}{c}\text { Test 2: } \\
\text { Comparaison doses-témoins }\end{array}$}} \\
\hline & & \multicolumn{3}{|c|}{ Doses } & \multicolumn{3}{|c|}{ Témoins } & & & & \\
\hline & & 0,2 & 0,4 & 0,8 & 1 & 2 & 3 & Valeur de $\mathrm{H}$ & Séparation & Valeur de $Z$ & Séparation \\
\hline 17 avril & $\begin{array}{l}0 \\
1 \\
2 \\
3 \\
4 \\
5\end{array}$ & $\begin{array}{r}36 \\
12 \\
2 \\
0 \\
0 \\
0\end{array}$ & $\begin{array}{r}45 \\
5 \\
0 \\
0 \\
0 \\
0\end{array}$ & $\begin{array}{r}42 \\
8 \\
0 \\
0 \\
0 \\
0\end{array}$ & $\begin{array}{r}36 \\
13 \\
1 \\
0 \\
0 \\
0\end{array}$ & $\begin{array}{r}44 \\
6 \\
0 \\
0 \\
0 \\
0\end{array}$ & $\begin{array}{r}26 \\
16 \\
8 \\
0 \\
0 \\
0\end{array}$ & $H^{\star}=31,11$ & $\stackrel{+}{+}(0,05$ et 0,01$)$ & $Z^{\star}=2,43$ & $\begin{array}{c}+ \\
(0,05 \text { et } 0,01)\end{array}$ \\
\hline 24 avril & $\begin{array}{l}0 \\
1 \\
2 \\
3 \\
4 \\
5\end{array}$ & $\begin{array}{r}17 \\
16 \\
14 \\
3 \\
0 \\
0\end{array}$ & $\begin{array}{r}37 \\
8 \\
5 \\
0 \\
0 \\
0\end{array}$ & $\begin{array}{r}25 \\
13 \\
11 \\
1 \\
0 \\
0\end{array}$ & $\begin{array}{r}26 \\
10 \\
9 \\
3 \\
2 \\
0\end{array}$ & $\begin{array}{r}29 \\
8 \\
6 \\
6 \\
0 \\
1\end{array}$ & $\begin{array}{r}14 \\
10 \\
13 \\
10 \\
3 \\
0\end{array}$ & $H=32,17$ & $\stackrel{+}{+}$ & $Z=2,27$ & $\stackrel{+}{(0,05)}$ \\
\hline 14 mai & $\begin{array}{l}0 \\
1 \\
2 \\
3 \\
4 \\
5\end{array}$ & $\begin{array}{r}14 \\
12 \\
14 \\
10 \\
0 \\
0\end{array}$ & $\begin{array}{r}28 \\
13 \\
8 \\
1 \\
0 \\
0\end{array}$ & $\begin{array}{r}21 \\
7 \\
13 \\
8 \\
1 \\
0\end{array}$ & $\begin{array}{r}14 \\
6 \\
8 \\
14 \\
8 \\
0\end{array}$ & $\begin{array}{r}26 \\
6 \\
8 \\
4 \\
5 \\
1\end{array}$ & $\begin{array}{r}9 \\
5 \\
9 \\
14 \\
13 \\
0\end{array}$ & $H=44,31$ & $\stackrel{+}{+} \stackrel{+}{(0,05}$ et 0,01$)$ & $Z=4,07$ & $\begin{array}{c}+ \\
(0,05 \\
\text { et } 0,01)\end{array}$ \\
\hline 22 juin & $\begin{array}{l}0 \\
1 \\
2 \\
3 \\
4 \\
5\end{array}$ & $\begin{array}{r}14 \\
11 \\
13 \\
10 \\
2 \\
0\end{array}$ & $\begin{array}{r}28 \\
13 \\
8 \\
1 \\
0 \\
0\end{array}$ & $\begin{array}{r}21 \\
7 \\
13 \\
8 \\
1 \\
0\end{array}$ & $\begin{array}{r}14 \\
6 \\
8 \\
14 \\
7 \\
1\end{array}$ & $\begin{array}{r}25 \\
5 \\
9 \\
3 \\
6 \\
2\end{array}$ & $\begin{array}{r}9 \\
4 \\
9 \\
14 \\
10 \\
4\end{array}$ & $H=44,07$ & $\stackrel{+}{+}$ & $Z=4,21$ & $\stackrel{+}{+}(0,05$ et 0,01$)$ \\
\hline 22 août & $\begin{array}{l}0 \\
1 \\
2 \\
3 \\
4 \\
5\end{array}$ & $\begin{array}{r}14 \\
11 \\
12 \\
11 \\
1 \\
1\end{array}$ & $\begin{array}{r}28 \\
13 \\
8 \\
1 \\
0 \\
0\end{array}$ & $\begin{array}{r}21 \\
7 \\
13 \\
8 \\
0 \\
1\end{array}$ & $\begin{array}{r}14 \\
6 \\
8 \\
14 \\
6 \\
2\end{array}$ & $\begin{array}{r}25 \\
5 \\
9 \\
3 \\
4 \\
4\end{array}$ & $\begin{array}{r}9 \\
4 \\
9 \\
14 \\
7 \\
7\end{array}$ & $H=43,75$ & $\stackrel{+}{+}(0,05$ et 0,01$)$ & $Z=4,14$ & $\stackrel{+}{+}$ \\
\hline
\end{tabular}

Test 1: test de Kruskall Wallis (valeur critique de la variable auxiliaire de Kuskall Wallis $H_{0}=11.07$ ) Test 2: test de Wilcoxon Mann Whitney (valeur critique de la variable $\mathrm{Z}: 1.6$ ).
" $\mathrm{H}=$ variable auxiliaire de Kruskall Wallis.

$Z$ = variable Wilcoxon sous la forme centrée réduite. 
relevé. Le test de Kruskall Wallis montre l'hétérogénéité des parcelles quelle que soit la date. La comparaison des parcelles traitées montre un effet de dose à partir du 24 avril, jusqu'au dernier relevé, la dose de $0,8 \mathrm{~g}$ de matière active par plant $(8 \mathrm{~g}$ de granulés) paraît assurer une bonne protection du plant.

D'autre part, la comparaison avec le traitement au Lindane montre une meilleure efficacité du Carbosulfan sur ce site, quelle que soit la dose. Ce résultat peut s'expliquer par le fait que les secteurs traités de manière standard (Lindane) se comportent, au bout de quelques semaines, comme les blocs témoins, le produit n'étant plus efficace contre l'Hylobe; il est alors nécessaire de répéter le traitement pour obtenir une protection efficace.

En 1988, le site de Lamazière-Basse a fait l'objet de quatre relevés, et seulement une très faible attaque a été détectée au début du printemps. Ainsi, il n'y a pas de modification significative par rapport aux résultats enregistrés en 1987.

Deux nouveaux relevés, au printemps et à l'automne 1989, compléteront les données sur l'efficacité du produit 24 mois après le traitement à la plantation.

Tableau IV Bilan des dégåts sur la parcelle expérimentale de Lamazière-Basse (19) 1988

\begin{tabular}{|c|c|c|c|c|c|c|c|c|c|c|c|}
\hline \multirow{3}{*}{$\begin{array}{l}\text { Oate de } \\
\text { relevé }\end{array}$} & \multirow{3}{*}{$\begin{array}{l}\text { Classes } \\
\text { de dégâts }\end{array}$} & \multicolumn{6}{|c|}{ Nombre d'arbres dans la classe } & \multirow{2}{*}{\multicolumn{2}{|c|}{$\begin{array}{l}\text { Test 1: } \\
\text { Comparaison des parcelles }\end{array}$}} & \multirow{2}{*}{\multicolumn{2}{|c|}{$\begin{array}{l}\text { Test 2: } \\
\text { Comparaison doses-témoins }\end{array}$}} \\
\hline & & \multicolumn{3}{|c|}{ Doses } & \multicolumn{3}{|c|}{ Témoins } & & & & \\
\hline & & 0,2 & 0,4 & 0,8 & 1 & 2 & 3 & Valeur de $\mathrm{H}$ & Séparation & Valeur de $Z$ & Séparation \\
\hline 23 avril & $\begin{array}{l}0 \\
1 \\
2 \\
3 \\
4 \\
5\end{array}$ & $\begin{array}{r}49 \\
0 \\
0 \\
0 \\
0 \\
1\end{array}$ & $\begin{array}{r}47 \\
2 \\
0 \\
0 \\
0 \\
1\end{array}$ & $\begin{array}{r}44 \\
1 \\
0 \\
0 \\
0 \\
4\end{array}$ & $\begin{array}{r}44 \\
3 \\
0 \\
0 \\
0 \\
3\end{array}$ & $\begin{array}{r}45 \\
0 \\
0 \\
0 \\
0 \\
5\end{array}$ & $\begin{array}{r}38 \\
1 \\
0 \\
0 \\
0 \\
8\end{array}$ & $H=12,18$ & $\stackrel{+}{(0,05)}$ & $Z=2,5$ & $\stackrel{+}{+}(0,05$ et 0,01$)$ \\
\hline 7 mai & $\begin{array}{l}0 \\
1 \\
2 \\
3 \\
4 \\
5\end{array}$ & $\begin{array}{r}48 \\
1 \\
0 \\
0 \\
0 \\
1\end{array}$ & $\begin{array}{r}47 \\
2 \\
0 \\
0 \\
0 \\
1\end{array}$ & $\begin{array}{r}44 \\
1 \\
1 \\
0 \\
0 \\
4\end{array}$ & $\begin{array}{r}44 \\
3 \\
0 \\
0 \\
0 \\
3\end{array}$ & $\begin{array}{r}45 \\
0 \\
0 \\
0 \\
0 \\
5\end{array}$ & $\begin{array}{r}38 \\
1 \\
3 \\
0 \\
0 \\
8\end{array}$ & $H=8,54$ & - & $Z=2,11$ & $\stackrel{+}{+}$ \\
\hline 5 juin & $\begin{array}{l}0 \\
1 \\
2 \\
3 \\
4 \\
5\end{array}$ & $\begin{array}{r}49 \\
0 \\
0 \\
0 \\
0 \\
1\end{array}$ & $\begin{array}{r}49 \\
0 \\
0 \\
0 \\
0 \\
1\end{array}$ & $\begin{array}{r}46 \\
0 \\
0 \\
0 \\
0 \\
4\end{array}$ & $\begin{array}{r}47 \\
0 \\
0 \\
0 \\
0 \\
3\end{array}$ & $\begin{array}{r}45 \\
0 \\
0 \\
0 \\
0 \\
5\end{array}$ & $\begin{array}{r}42 \\
0 \\
0 \\
0 \\
0 \\
8\end{array}$ & $H=8,54$ & - & $Z=2,11$ & $\stackrel{+}{+}$ \\
\hline $\begin{array}{l}5 \text { sep- } \\
\text { tembre }\end{array}$ & $\begin{array}{l}0 \\
1 \\
2 \\
3 \\
4 \\
5\end{array}$ & $\begin{array}{r}48 \\
0 \\
0 \\
0 \\
0 \\
1\end{array}$ & $\begin{array}{r}48 \\
0 \\
0 \\
0 \\
0 \\
1\end{array}$ & $\begin{array}{r}46 \\
0 \\
0 \\
0 \\
0 \\
4\end{array}$ & $\begin{array}{r}47 \\
0 \\
0 \\
0 \\
0 \\
3\end{array}$ & $\begin{array}{r}45 \\
0 \\
0 \\
0 \\
0 \\
5\end{array}$ & $\begin{array}{r}42 \\
0 \\
0 \\
0 \\
0 \\
8\end{array}$ & $H=8,32$ & - & $Z=2,08$ & $\stackrel{+}{+}$ \\
\hline $\begin{array}{l}24 \\
\text { octobre }\end{array}$ & $\begin{array}{l}0 \\
1 \\
2 \\
3 \\
4 \\
5\end{array}$ & $\begin{array}{r}48 \\
0 \\
0 \\
0 \\
0 \\
1\end{array}$ & $\begin{array}{r}48 \\
0 \\
0 \\
0 \\
0 \\
1\end{array}$ & $\begin{array}{r}46 \\
0 \\
0 \\
0 \\
0 \\
4\end{array}$ & $\begin{array}{r}47 \\
0 \\
0 \\
0 \\
0 \\
3\end{array}$ & $\begin{array}{r}45 \\
0 \\
0 \\
0 \\
0 \\
5\end{array}$ & $\begin{array}{r}42 \\
0 \\
0 \\
0 \\
0 \\
8\end{array}$ & $H=8,32$ & - & $Z=2,08$ & $\stackrel{+}{+}$ \\
\hline
\end{tabular}




\section{- Pérols-sur-Vézère}

En 1987, nous n'avons observé qu'une très faible attaque d'Hylobes à la fin de l'été. Les différents tests ne permettent pas de déceler une hétérogénéité quelles que soient les comparaisons effectuées. Néanmoins, à partir du 14 mai, le test de Wilcoxon Mann Whitney permet de séparer les parcelles traitées des parcelles témoins.

En 1988, de fin avril à fin octobre, nous avons assisté à une très forte attaque d'Hylobes, qui s'est déroulée en deux vagues successives:

- de début avril à fin juin, avec des insectes sortant d'hivernation ;

- de début septembre à fin octobre, avec ces mêmes insectes, les insectes issus de larves ayant hiveriné et des insectes issus de la ponte de printemps.

Les différents tests appliqués aux résultats montre une bonne séparation entre parcelles traitées et parcelles témoins, ainsi qu'un effet de dose très net, la parcelle traitée à $8 \mathrm{~g}$ de Carbosulfan

Tableau V Bilan des dégâts sur la parcelle expérimentale de Pérols-sur-Vézère (19) 1987

\begin{tabular}{|c|c|c|c|c|c|c|c|c|c|c|c|}
\hline \multirow{3}{*}{$\begin{array}{l}\text { Date de } \\
\text { relevé }\end{array}$} & \multirow{3}{*}{$\begin{array}{l}\text { Classes } \\
\text { de dégâts }\end{array}$} & \multicolumn{6}{|c|}{ Nombre d'arbres dans la classe } & \multirow{2}{*}{\multicolumn{2}{|c|}{$\begin{array}{l}\text { Test 1: } \\
\text { Comparaison des parcelles }\end{array}$}} & \multirow{2}{*}{\multicolumn{2}{|c|}{$\begin{array}{l}\text { Test } 2 \text { : } \\
\text { Comparaison doses-témoins }\end{array}$}} \\
\hline & & \multicolumn{3}{|c|}{ Doses } & \multicolumn{3}{|c|}{ Témoins } & & & & \\
\hline & & 0,2 & 0,4 & 0,8 & 1 & 2 & 3 & Valeur de $\mathrm{H}$ & Séparation & Valeur de Z & Séparation \\
\hline 17 avril & $\begin{array}{l}0 \\
1 \\
2 \\
3 \\
4 \\
5\end{array}$ & $\begin{array}{r}50 \\
0 \\
0 \\
0 \\
0 \\
0\end{array}$ & $\begin{array}{r}49 \\
1 \\
0 \\
0 \\
0 \\
0\end{array}$ & $\begin{array}{r}50 \\
0 \\
0 \\
0 \\
0 \\
0\end{array}$ & $\begin{array}{r}50 \\
0 \\
0 \\
0 \\
0 \\
0\end{array}$ & $\begin{array}{r}50 \\
0 \\
0 \\
0 \\
0 \\
0\end{array}$ & $\begin{array}{r}50 \\
0 \\
0 \\
0 \\
0 \\
0\end{array}$ & $H=4,99$ & - & $z=1$ & - \\
\hline 24 avril & $\begin{array}{l}0 \\
1 \\
2 \\
3 \\
4 \\
5\end{array}$ & $\begin{array}{r}50 \\
0 \\
0 \\
0 \\
0 \\
0\end{array}$ & $\begin{array}{r}48 \\
2 \\
0 \\
0 \\
0 \\
0\end{array}$ & $\begin{array}{r}50 \\
0 \\
0 \\
0 \\
0 \\
0\end{array}$ & $\begin{array}{r}48 \\
2 \\
0 \\
0 \\
0 \\
0\end{array}$ & $\begin{array}{r}50 \\
0 \\
0 \\
0 \\
0 \\
0\end{array}$ & $\begin{array}{r}48 \\
2 \\
0 \\
0 \\
0 \\
0\end{array}$ & $H=6,10$ & - & $Z=0,82$ & - \\
\hline 14 mai & $\begin{array}{l}0 \\
1 \\
2 \\
3 \\
4 \\
5\end{array}$ & $\begin{array}{r}50 \\
0 \\
0 \\
0 \\
0 \\
0\end{array}$ & $\begin{array}{r}47 \\
2 \\
1 \\
0 \\
0 \\
0\end{array}$ & $\begin{array}{r}48 \\
2 \\
0 \\
0 \\
0 \\
0\end{array}$ & $\begin{array}{r}46 \\
3 \\
1 \\
0 \\
0 \\
0\end{array}$ & $\begin{array}{r}47 \\
3 \\
0 \\
0 \\
0 \\
0\end{array}$ & $\begin{array}{r}44 \\
5 \\
0 \\
1 \\
0 \\
0\end{array}$ & $H=7,08$ & - & $Z=1,94$ & $\stackrel{+}{(0,05)}$ \\
\hline 22 juin & $\begin{array}{l}0 \\
1 \\
2 \\
3 \\
4 \\
5\end{array}$ & $\begin{array}{r}50 \\
0 \\
0 \\
0 \\
0 \\
0\end{array}$ & $\begin{array}{r}47 \\
2 \\
1 \\
0 \\
0 \\
0\end{array}$ & $\begin{array}{r}48 \\
2 \\
0 \\
0 \\
0 \\
0\end{array}$ & $\begin{array}{r}46 \\
3 \\
1 \\
0 \\
0 \\
0\end{array}$ & $\begin{array}{r}47 \\
3 \\
0 \\
0 \\
0 \\
0\end{array}$ & $\begin{array}{r}44 \\
5 \\
0 \\
1 \\
0 \\
0\end{array}$ & $H=7,08$ & - & $Z=1,94$ & $\stackrel{+}{(0,05)}$ \\
\hline 22 août & $\begin{array}{l}0 \\
1 \\
2 \\
3 \\
4 \\
5\end{array}$ & $\begin{array}{r}41 \\
6 \\
3 \\
0 \\
0 \\
0\end{array}$ & $\begin{array}{r}41 \\
4 \\
5 \\
0 \\
0 \\
0\end{array}$ & $\begin{array}{r}41 \\
8 \\
1 \\
0 \\
0 \\
0\end{array}$ & $\begin{array}{r}37 \\
10 \\
2 \\
3 \\
0 \\
0\end{array}$ & $\begin{array}{r}36 \\
6 \\
3 \\
5 \\
0 \\
0\end{array}$ & $\begin{array}{r}29 \\
6 \\
3 \\
1 \\
1 \\
0\end{array}$ & $H=3,58$ & - & $Z=1,68$ & $\stackrel{+}{+}$ \\
\hline
\end{tabular}


par plant $(0,8 \mathrm{~g}$ de matière active) assurant une excellente protection 20 mois après le traitement appliqué en avril 1987.

D'autre part, lorsque l'on effectue une comparaison entre le traitement standard (à raison de $360 \mathrm{~g}$ de matière active à l'hectare) et les différentes doses de Carbosulfan employées dans l'essai, les doses de 2 et $4 \mathrm{~g}$ de Carbosulfan par plant (respectivement $0,2 \mathrm{~g}$ et $0,4 \mathrm{~g}$ de matière active) donnent des résultats équivalents; par contre, on peut noter une différence très significative avec la dose de $8 \mathrm{~g}$ de Carbosulfan par plant qui assure une protection nettement supérieure en un seul traitement.

Tableau VI Bilan des dégâts sur la parcelle expérimentale de Pérols-sur-Vézère (19) 1988

\begin{tabular}{|c|c|c|c|c|c|c|c|c|c|c|c|}
\hline \multirow{3}{*}{$\begin{array}{l}\text { Dafe de } \\
\text { relevé }\end{array}$} & \multirow{3}{*}{$\begin{array}{l}\text { Classes } \\
\text { de dégâts }\end{array}$} & \multicolumn{6}{|c|}{ Nombre d'arbres dans la classe } & \multirow{2}{*}{\multicolumn{2}{|c|}{$\begin{array}{l}\text { Test 1: } \\
\text { Comparaison des parcelles }\end{array}$}} & \multirow{2}{*}{\multicolumn{2}{|c|}{$\begin{array}{l}\text { Test 2: } \\
\text { Comparaison doses-fémoins }\end{array}$}} \\
\hline & & \multicolumn{3}{|c|}{ Doses } & \multicolumn{3}{|c|}{ Témoins } & & & & \\
\hline & & 0,2 & 0,4 & 0,8 & 1 & 2 & 3 & Valeur de $\mathrm{H}$ & Séparation & Valeur de $Z$ & Séparation \\
\hline 23 avril & $\begin{array}{l}0 \\
1 \\
2 \\
3 \\
4 \\
5\end{array}$ & $\begin{array}{r}3 \\
5 \\
10 \\
13 \\
18 \\
1\end{array}$ & $\begin{array}{r}8 \\
4 \\
11 \\
13 \\
11 \\
3\end{array}$ & $\begin{array}{r}8 \\
13 \\
16 \\
12 \\
1 \\
0\end{array}$ & $\begin{array}{r}2 \\
2 \\
8 \\
16 \\
21 \\
1\end{array}$ & $\begin{array}{r}4 \\
4 \\
5 \\
11 \\
20 \\
6\end{array}$ & $\begin{array}{r}4 \\
6 \\
13 \\
13 \\
13 \\
1\end{array}$ & $H=42,08$ & $(0,05 \stackrel{+}{\text { ef }} 0,01)$ & $Z=3,95$ & $(0,05$ et 0,01$)$ \\
\hline 7 mai & $\begin{array}{l}0 \\
1 \\
2 \\
3 \\
4 \\
5\end{array}$ & $\begin{array}{r}1 \\
2 \\
3 \\
21 \\
20 \\
3\end{array}$ & $\begin{array}{r}0 \\
6 \\
3 \\
21 \\
16 \\
4\end{array}$ & $\begin{array}{r}0 \\
3 \\
18 \\
26 \\
2 \\
0\end{array}$ & $\begin{array}{r}0 \\
1 \\
1 \\
22 \\
21 \\
5\end{array}$ & $\begin{array}{r}2 \\
0 \\
6 \\
14 \\
20 \\
8\end{array}$ & $\begin{array}{r}0 \\
2 \\
14 \\
17 \\
10 \\
7\end{array}$ & $H=40,27$ & $(0,05 \stackrel{+}{\text { et }} 0,01)$ & $Z=3,04$ & $(0,05$ et 0,01$)$ \\
\hline 5 juin & $\begin{array}{l}0 \\
1 \\
2 \\
3 \\
4 \\
5\end{array}$ & $\begin{array}{r}1 \\
2 \\
3 \\
21 \\
20 \\
3\end{array}$ & $\begin{array}{r}0 \\
6 \\
3 \\
21 \\
16 \\
4\end{array}$ & $\begin{array}{r}0 \\
4 \\
18 \\
26 \\
2 \\
0\end{array}$ & $\begin{array}{r}0 \\
1 \\
1 \\
22 \\
21 \\
5\end{array}$ & $\begin{array}{r}2 \\
0 \\
6 \\
14 \\
20 \\
8\end{array}$ & $\begin{array}{r}0 \\
2 \\
14 \\
17 \\
10 \\
7\end{array}$ & $H=41,53$ & $(0,05 \stackrel{+}{\text { ef }} 0,01)$ & $Z=3,23$ & $(0,05 \stackrel{+}{\text { et }} 0,01)$ \\
\hline $\begin{array}{l}5 \text { sep- } \\
\text { tembre }\end{array}$ & $\begin{array}{l}0 \\
1 \\
2 \\
3 \\
4 \\
5\end{array}$ & $\begin{array}{r}13 \\
12 \\
18 \\
0 \\
0 \\
7\end{array}$ & $\begin{array}{r}27 \\
15 \\
3 \\
0 \\
0 \\
5\end{array}$ & $\begin{array}{r}7 \\
18 \\
16 \\
4 \\
0 \\
0\end{array}$ & $\begin{array}{r}3 \\
28 \\
8 \\
0 \\
0 \\
11\end{array}$ & $\begin{array}{r}10 \\
9 \\
19 \\
1 \\
0 \\
11\end{array}$ & $\begin{array}{r}0 \\
35 \\
5 \\
0 \\
0 \\
10\end{array}$ & $H=30,82$ & $\stackrel{+}{+}+\underset{+}{0,05}$ et 0,01$)$ & $Z=3,64$ & $(0,05$ et 0,01$)$ \\
\hline $\begin{array}{l}25 \\
\text { octobre }\end{array}$ & $\begin{array}{l}0 \\
1 \\
2 \\
3 \\
4 \\
5\end{array}$ & $\begin{array}{r}5 \\
5 \\
8 \\
19 \\
5 \\
8\end{array}$ & $\begin{array}{r}0 \\
2 \\
5 \\
33 \\
4 \\
6\end{array}$ & $\begin{array}{r}0 \\
7 \\
10 \\
27 \\
1 \\
0\end{array}$ & $\begin{array}{r}0 \\
2 \\
4 \\
27 \\
5 \\
12\end{array}$ & $\begin{array}{r}6 \\
2 \\
7 \\
15 \\
7 \\
13\end{array}$ & $\begin{array}{r}0 \\
1 \\
10 \\
19 \\
7 \\
12\end{array}$ & $H=19,59$ & $\stackrel{+}{+}+(0,05$ et 0,01$)$ & $Z=3,29$ & $(0,05$ et 0,01$)$ \\
\hline $\begin{array}{l}12 \\
\text { décembre }\end{array}$ & $\begin{array}{l}0 \\
1 \\
2 \\
3 \\
4 \\
5\end{array}$ & $\begin{array}{r}41 \\
0 \\
0 \\
0 \\
0 \\
9\end{array}$ & $\begin{array}{r}44 \\
0 \\
0 \\
0 \\
0 \\
6\end{array}$ & $\begin{array}{r}45 \\
0 \\
0 \\
0 \\
0 \\
0\end{array}$ & $\begin{array}{r}38 \\
0 \\
0 \\
0 \\
0 \\
12\end{array}$ & $\begin{array}{r}35 \\
0 \\
0 \\
0 \\
0 \\
15\end{array}$ & $\begin{array}{r}36 \\
0 \\
0 \\
0 \\
0 \\
13\end{array}$ & $H=18,87$ & $(0,05 \stackrel{+}{\text { ef }} 0,01)$ & $Z=3,62$ & $(0,05$ et 0,01$)$ \\
\hline
\end{tabular}

Nota : A partir du 5 septembre les relevés sur la parcelle traitée à 0,8 gramme de matiére active par plant portent sur 45 sujets. 5 sujets ont été prélevés pour analyse à I'ENSA de Toulouse.

De méme pour le témoin 3, dont les relevés portent sur 49 sujets à partir du 25 octobre, le sujet manquant ayant succombe à une attaque d'Armillaire. 

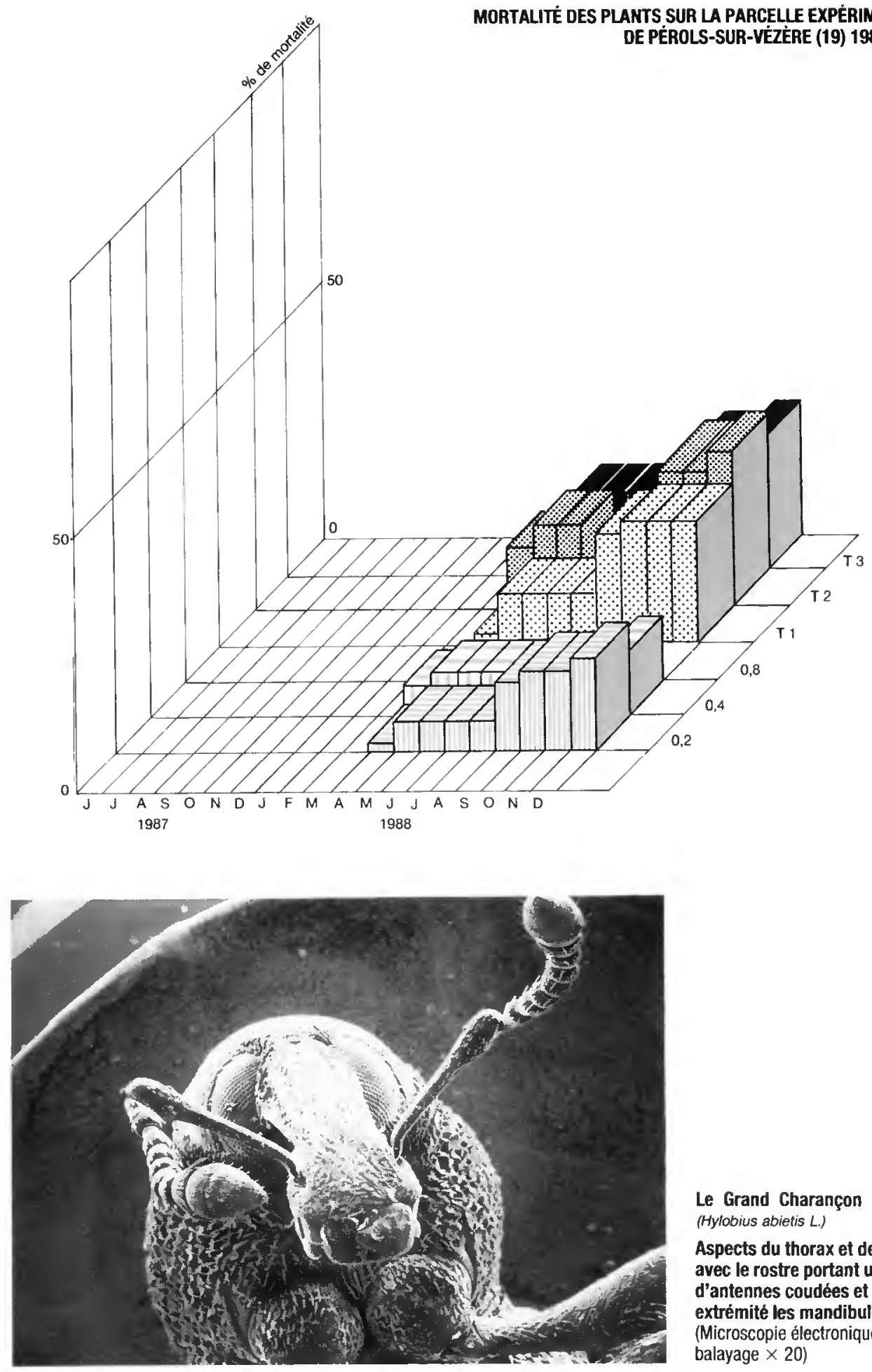

Le Grand Charançon du Pin (Hylobius abietis L.)

Aspects du thorax et de la tête avec le rostre portant une paire d'antennes coudées et à son extrèmité les mandibules. (Microscopie électronique à balayage $\times 20$ ) 
En 1988, les essais de terrain ont été étendus à quatre nouveaux sites en Limousin-Auvergne, quatre sites dans le Morvan, et un site en Bretagne. Les différentes observations permettront d'affiner les résultats.

\section{CONCLUSIONS}

Ces tests préliminaires laissent entrevoir qu'une bonne protection peut être attendue grâce au Carbosulfan. Ils doivent être poursuivis afin de vérifier la protection à long terme des plants.

Enfin, des traitements en pépinière permettront de vérifier si une protection complète des plants peut être obtenue. Une question délicate est liée au décalage (une semaine environ) entre le traitement du plant et la remontée de l'insecticide dans le végétal, l'Hylobe pouvant occasionner de graves dégâts pendant ce laps de temps. Comme nous l'avons précédemment souligné, en réduisant le nombre de traitements à un seul, en étant sans danger pour l'utilisateur, en offrant une technologie alternative acceptable pour l'environnement et permettant de remplacer l'emploi des pesticides organochlorés, cette nouvelle formulation offre des bénéfices certains tant sur le plan économique qu'écologique.

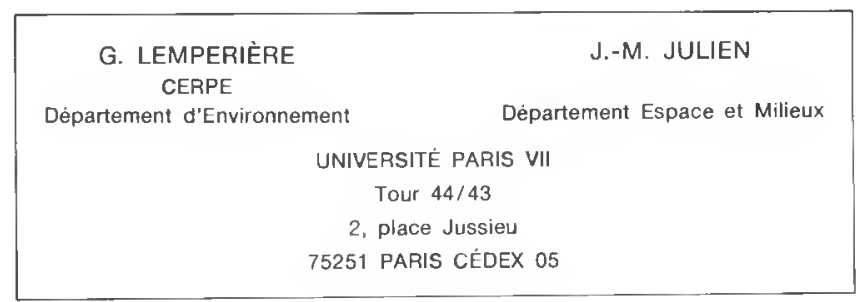

Remerciements : Les auteurs tiennent à remercier Messieurs Lorain, Geering, Bevan, Descoins et Malphettes pour leurs remarques critiques et leur aide dans la mise en forme de cet article, ainsi que les personnels du Service forestier de la Direction départementale de l'Agriculture el de la Forêt de Corréze pour leur aide sur le terrain.

Nota : Le Marshal Suxon a reçu une autorisation provisoire de vente $\left(n^{\circ} 8800255\right)$ délivrée en décembre 1988 pour une dose d'emploi de $10 \mathrm{~g}$ (un gramme de matière active) par plant, en traitement du sol.

\section{BIBLIOGRAPHIE}

BAKKE (A.), CHRISTIANSEN (E.). - Testing insecticides against the Pine Weevil (Hylobius abietis L.), Proceedings of the 6th British Insecticide Conference, 1971, pp. 808-816.

CEMAGREF. - Intormation technique pour la surveillance et la protection phytosanitaire de la forèt ; tascicule II : fiche sur le Grand Charençon du Pin, Hylobius abietis L.. - CEMAGREF, 1975.

CEMAGREF. - Essais de lutte contre l'Hylobe menés en 1984 dans les plantations résineuses de RhôneAlpes, Auvergne et Limousin. - CEMAGREF-Division Protection de la Nature, 1984. - $9 p$. 
DELORME (R.), MALPHETTES (C.-B.). - Rèsultats préliminaires de tests de laboratoire effectués pour évaluer l'etficacite de divers insecticides contre l'Hylobe (Hylobius abietis L., Coleoptera, Curculionidae). Phytiatrie-Phytopharmacie, 25, 1976, pp. 123-130.

GARNER (W.) et al. - Evaluation of pesticides in ground water. - ACS Symposium series, $1986,315$.

KING (C.J.), SCOTT (T.N.). - The large Pine Weevil and Black Pine Beetles. - Forestry Commission Leaflet, $52,1974,12 \mathrm{p}$.

LEMPERIĖRE (G.). - Early investigations on the potential of alternative prey for the rearing of Rhizophagus grandis, predatory beetle of Dendroctonus micans (Col. Scolytidae), the Great Spruce Bark Beetle. Proceeding of a seminar organized by the Commission of the European Communities and the Université Libre de Bruxelles, 1984, pp. 129-133.

MAY (P.D.), BOEHM (N.). - Controlled release soil insecticides for control of sugar cane pests; Sugar $y$ azucar. - July-August 1986, pp. 127-132.

PLOWMAN (N.), O'HANLON (G.J.). - Controlled release formulations of soil applied pesticides. - Proceeding of the 4th Australian Applied Entomological Research Conference, Adelaide, 1984.

TILLES (D.A.), NORDLANDFR (G.), NORDENHEM (H.), EIDMANN (H.H.), WASSGREN (A.), BERGSTROM (G.). Increased release of host volatiles from feeding scars: a major cause of field aggregation in the Pine Weevil Hylobius abietis (Coleoptera: Curculionidae). - Environmental Entomology, vol. 15, $n^{\circ} 5,1986$, pp. 1050-1054.

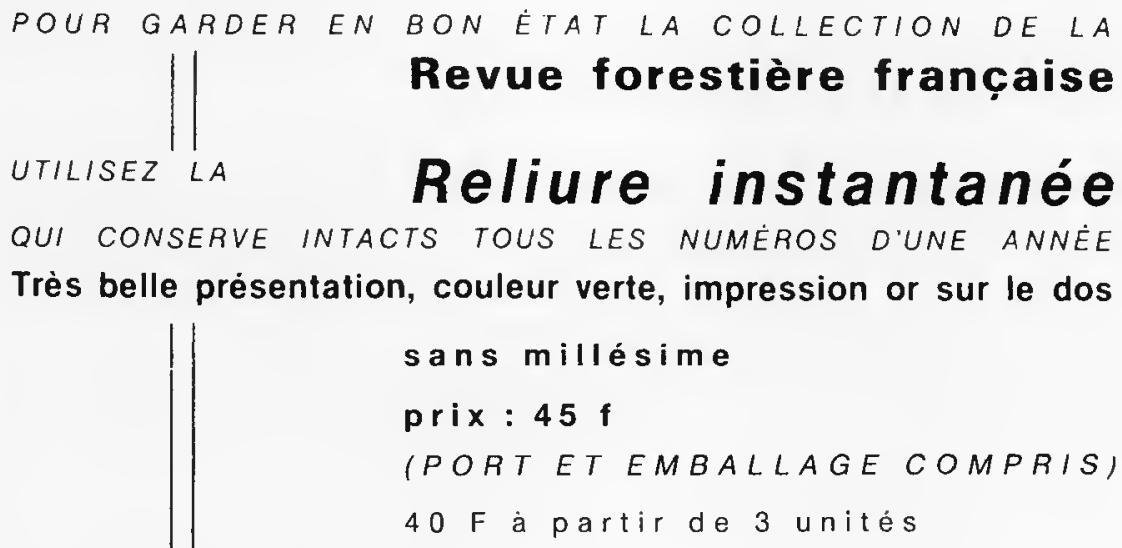

Très belle présentation, couleur verte, impression or sur le dos

sans millésime

prix : 45 f

(PORT ET EMBALLAGE COMPRIS)

$40 \mathrm{~F}$ à partir de 3 unités

Les commandes sont à envoyer à l'adresse suivante :

Revue forestière française $\cdot 14$, rue Girardet, 54042 NANCY CEDEX

Paiement au C. C. P. Nancy 5.400.64 D

au nom du Régisseur de l'École Nationale du Génie Rural des Eaux et des Forêts 